\title{
A Search for Charged Massive Stable Particles
}

\author{
Thomas Nunnemann*† \\ LMU Munich, Germany \\ E-mail: Thomas.Nunnemann@physik. uni-muenchen. de
}

\begin{abstract}
A search for charged massive (quasi-) stable particles with the DØ detector at the Tevatron collider based on $390 \mathrm{pb}^{-1}$ of data is presented. The search is performed in the frameworks of gauge-mediated supersymmetry breaking and the minimal supersymmetric extentension of the standard model. The hypothetical particles are assumed to be pair-produced in $p \bar{p}$ collisions giving a signature of two reconstructed muon-like objects with high invariant mass and time-offlights indicative of heavy particles. Since no excess over background is observed, cross-section limits for the pair-production of stable staus and charginos are set. Mass limits of $140 \mathrm{GeV}$ for a higgsino-like chargino and $174 \mathrm{GeV}$ for a gaugino-like chargino are set.
\end{abstract}

International Europhysics Conference on High Energy Physics

July 21st - 27th 2005

Lisboa, Portugal

\footnotetext{
*Speaker.

† for the DØ Collaboration
} 


\section{Introduction}

We report on the search for new charged massive stable particles (CMSP) assumed to be pairproduced in $p \bar{p}$ collisions. Herein, stable refers to a lifetime long enough to escape the entire detector without decaying. Heavy particles can be identified with the $\mathrm{D} \emptyset$ detector primarily using the time-of-flight measurement of the muon detector's scintillators, which have a time resolution of 2-3 ns.

Several supersymmetric (SUSY) models can include a long-lived, quasi-stable particle as nextto-lightest supersymmetric particle (NLSP) provided that it is nearly mass-degenerate with the lightest supersymmetric particle (LSP) or that its coupling to the LSP is small.

The latter condition can be fulfilled in models with gauge-mediated supersymmetry breaking (GMSB), where gauge interactions with messenger fields at a scale much smaller than the Planck scale are responsible for the SUSY breaking [1]. GMSB models have a very distinctive phenomenology. The gravitino $\tilde{G}$ is typically light $(\lesssim 1 \mathrm{keV})$ and is the lightest SUSY particle (LSP). The next-to-lightest SUSY particle (NLSP) is usually either the lightest neutralino $\tilde{\chi}_{1}^{0}$, decaying into $\gamma \tilde{G}$, or the lightest charged slepton (mostly $\tilde{\tau}_{1}$ ), decaying into $l \tilde{G}$. Due to the weak gravitational coupling the NLSP can be quasi-stable. In this analysis, the GMSB model referred to as "Snowmass Model Line D" which contains a stau as NLSP is used [3]. Its parameter set is shown in Table 1.

In anomaly-mediated supersymmetry breaking models the lightest chargino $\tilde{\chi}_{1}^{ \pm}$(NLSP) is nearly mass-degenerate with the lightest neutralino $\tilde{\chi}_{1}^{0}$ (LSP) [2]. Two general cases have been studied in this analysis in the framework of the MSSM (see Table 2) [4]: higgsino-like $\tilde{\chi}_{1}^{ \pm}, \tilde{\chi}_{1}^{0}$ (deep-higgsino region) and gaugino-like $\tilde{\chi}_{1}^{ \pm}, \tilde{\chi}_{1}^{0}$ (AMSB inspired).

For both the GMSB and chargino analysis, signal events were generated with Pythia 6.202 [5] and passed through a parameterized Monte Carlo simulation, which includes all efficiencies and detector resolution smearing. In particular the muon system's time measurements are smeared according to resolutions and offsets measured in data. Only the pair-production of the lightest $\tilde{\tau}$ or the chargino, respectively, is considered. However, the analysis has also some sensitivity to CMSPs produced in cascade-decays of heavier supersymmetric particles.

The data sample consists of $390 \mathrm{pb}^{-1}$. The trigger employed required two tracks in the muon system using an asymmetric trigger gate to be efficient for particles travelling slower than light. Nevertheless the trigger efficiency drops to about $75 \%$ for $M_{\tilde{\tau}}=300 \mathrm{GeV}$.

\begin{tabular}{lcccccc}
\hline \hline Model & $\Lambda_{m}[\mathrm{GeV}]$ & $M_{m}$ & $N_{5}$ & $\tan \beta$ & $\operatorname{sgn} \mu$ & $C_{\text {grav }}$ \\
\hline GMSB “Model Line D” & from 19 to $100 \mathrm{TeV}$ & $2 \Lambda_{m}$ & 3 & 15 & +1 & 1 \\
\hline \hline
\end{tabular}

Table 1: GMSB “Model Line D” parameter

\begin{tabular}{lcccccc}
\hline \hline Model & $\mu[\mathrm{GeV}]$ & $M_{1}[\mathrm{GeV}]$ & $M_{2}[\mathrm{GeV}]$ & $M_{3}[\mathrm{GeV}]$ & $\tan \beta$ & $M_{\tilde{q}}[\mathrm{GeV}]$ \\
\hline higgsino-like & from 60 to 300 & 100,000 & 100,000 & 500 & 15 & 800 \\
gaugino-like & 10,000 & $3 M_{2}$ & from 60 to 300 & 500 & 15 & 800 \\
\hline \hline
\end{tabular}

Table 2: MSSM parameter sets for chargino analysis 

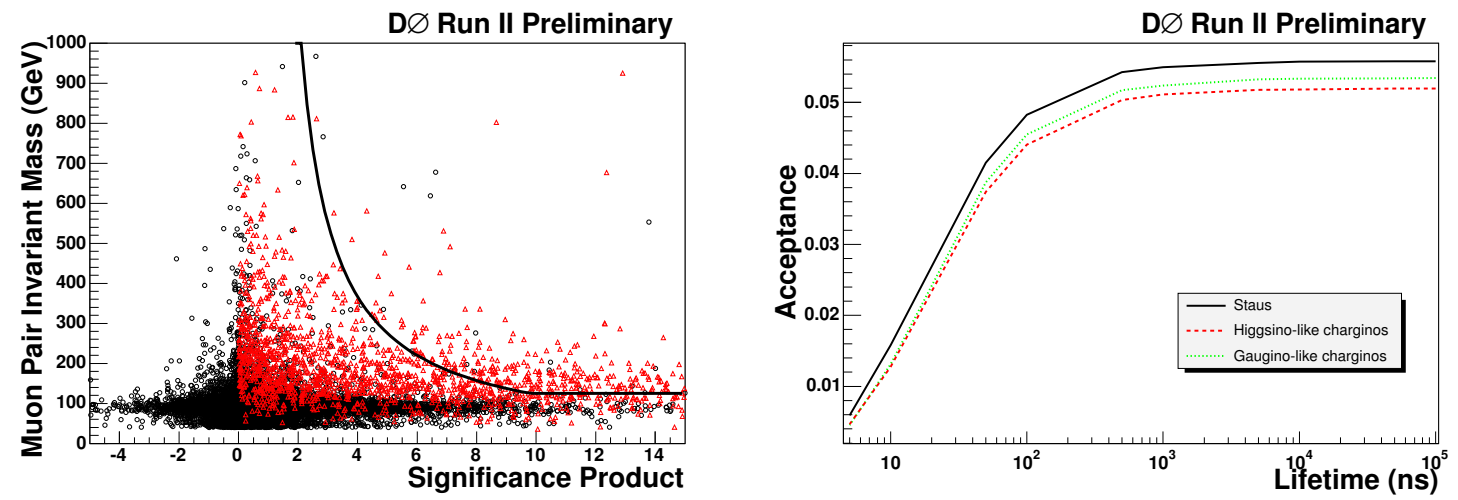

Figure 1: Left: Invariant mass versus significance product for reconstructed muon pairs in data (black circles) and stau pairs with $M_{\tilde{\tau}}=60 \mathrm{GeV}$ (red triangles). The line represents the optimized two-dimensional cut. Right: Acceptance versus lifetime for assumed $M_{\tilde{\tau}}=100 \mathrm{GeV}$.

\section{Selection and Background Estimation}

The analysis requires two reconstructed muons with transverse momentum $p_{T}>15 \mathrm{GeV}$, both matched to a central track and with hits in at least two of the three scintillator layers. Cosmic veto cuts are applied and at least one muon is required to be isolated to suppress background from heavyflavor production. To ensure a good speed measurement for each particle in the event, consistent time information in all scintillator layers are required. No separation with a $\mathrm{d} E / \mathrm{d} x$ measurement in the central fiber tracker is attempted due to its small number of layers and small photon statistics from scintillation. After this preselection the background is largely dominated by $Z$ and Drell-Yan production.

To quantify the deviation of a particle's speed $v$ from the speed of light $c$, a speed significance is defined as $(c-v) / \sigma_{v}$ which is required to be positive. The final signal selection is based on a twodimensional cut using the invariant di-muon mass $M_{\mu \mu}$ and the product of the speed significance of the two reconstructed muons (CMSP candidates). This cut is optimized separately for each CMSP mass hypothesis (see Fig. 1, left). Since the kinematic properties of the considered signal models are similar, the optimization obtained with the GMSB model is used for the other models as well.

The background estimation is entirely based on data using orthogonal data sets to describe the two-dimensional probability density function (PDF) depending on the invariant di-muon mass and the significance product. Since no correlation between these two variables is observed for the background, which is predominantly $Z$ and Drell-Yan production, the PDF can be factorized. Events with apparent muon speeds $v_{\mu_{1,2}}>c$ are used to estimate the background's invariant di-muon mass distribution and events with $M_{\mu \mu} \approx M_{Z}$ are taken to estimate the significance product.

The systematic error on the signal acceptance is dominated by uncertainties in the trigger, muon identification and timing cut efficiencies as implemented in the simulation, and amounts to $2.7 \%$. The systematic error on the background prediction has been evaluated to be $3.7 \%$ by varying the selection criteria. 

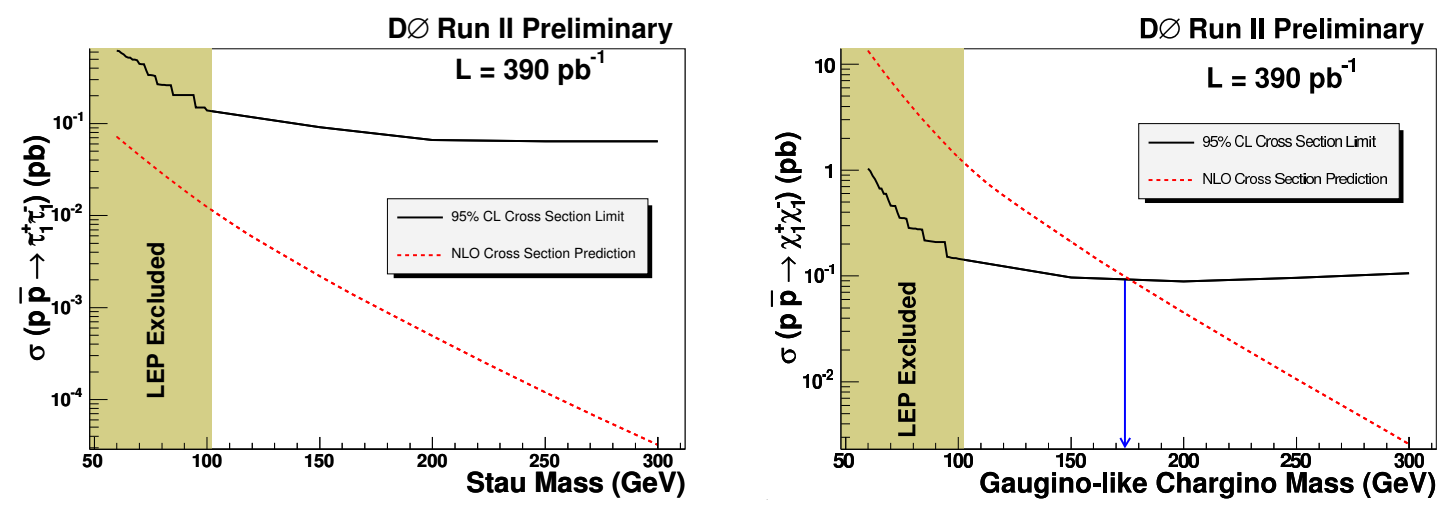

Figure 2: 95\% cross-section limit (solid line) and NLO production cross-section for pair-produced staus in GMSB (left) and gaugino-like charginos (right).

\section{Results and Conclusions}

For both the GMSB and the chargino analysis, six CMSP mass points from 60 to $300 \mathrm{GeV}$ have been studied. The data are consistent with the background estimation. For assumed CMSP masses larger than $100 \mathrm{GeV}$, no events are observed, with background predictions ranging from 0.5 to 0.7 events. Signal acceptances are between $2.3 \%$ and $12.3 \%$ depending on CMSP mass and model. For finite CMSP life-times the acceptance decreases as shown in Fig. 1, right.

95\% confidence level limits on the cross-section for CMSP pair-production are set using the $C L_{S}$ method [6] for each model and CMSP mass point and are subsequently compared to the nextto-leading order cross-section predictions calculated with Prospino 2 [7]. In the case of the GMSB analysis, these limits are not yet stringent enough to set a limit on the stau mass, nevertheless they are the best limits to date from the Tevatron (cf. Fig.2, left).

The cross-section limits for the chargino-analyses are shown in Fig.2, right. Mass limits for the stable chargino could be set beyond the LEP exclusion limit of $102.5 \mathrm{GeV}$ [8]: For higgsino-like stable charginos masses below $140 \mathrm{GeV}$, for gaugino-like stable charginos masses below $174 \mathrm{GeV}$ are excluded. These are currently the best mass limits on stable charginos.

\section{References}

[1] For a review see: G.F. Giudice and R. Rattazzi, Phys. Rept. 322, (1999) 419.

[2] L. Randall and R. Sundrum, Nucl. Phys. B557, (1999) 79.

[3] S. Martin et. al, FERMILAB-CONF-01-371-T.

[4] For a review see: S. Martin, in Kane, G.(ed.): Perspectives on supersymmetry 1-98, 1997.

[5] T. Sjosstrand et.al., Computer Physics Comun. 135, 238 (2001).

[6] T. Junk, Nucl. Instrum. Methods A 434, 435 (1999).

[7] W. Beenakker et. al., Phys. Rev. Lett. 83, 3780 (1999).

[8] LEP SUSY Work. Group, http: / / lepsusy.web. cern. ch. 